Divers@ Revista Eletrônica Interdisciplinar,

Matinhos, v. 13, n. 2, p. 154-163, jul./dez. 2020

ISSN 1983-8921

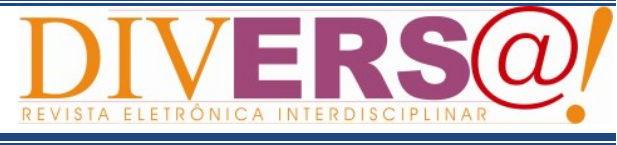

\title{
SMARTS CITIES, SAÚDE E SUSTENTABILIDADE: UMA BREVE REVISÃO
}

\author{
SMARTS CITIES, HEALTH AND SUSTAINABILITY: A BRIEF REVIEW
}

\author{
Daniela Alves Cardeal dos Santos ${ }^{1}$ \\ Augusto Junior Clemente ${ }^{2}$ \\ Márcia Rejane Chitolina Perini ${ }^{3}$ \\ Iris Nabolotnyj Martinez ${ }^{4}$ \\ Caroline Santos da Rosa ${ }^{5}$
}

\begin{abstract}
Resumo
A terminologia "cidades inteligentes" foi incorporada pelas Tecnologias da Informação e da Comunicação (TICs) para direcionar indicadores sobre o impacto de uma cidade "conectada" com as pessoas. O presente ensaio visa apresentar uma reflexão sobre esta temática com o enfoque sobre os aspectos da saúde pública e do desenvolvimento sustentável que permeiam a efetivação de políticas públicas adequadas à promoção da saúde e qualidade de vida nos cenários das comunidades contemporâneas. A relevância desse estudo contribui para a reflexão, a motivação e a mobilização de subsídios práticos e teóricos sobre as cidades, motivando o uso adequado dos recursos econômicos, combinados com o bem-estar da vida dos habitantes e com a diminuição da emissão de poluentes, o que corresponde a efetivação de políticas públicas voltadas às necessidades de melhoria de vida da população.
\end{abstract}

Palavras-chave: Tecnologias da Informação e da Comunicação (TICs); Desenvolvimento Sustentável; Promoção da Saúde Pública.

\begin{abstract}
The terminology "smart cities" has been incorporated by Information and Communication Technologies (ICTs) to target indicators on the impact about city "connected" with people. This essay aims to present a reflection on this theme with a focus on the aspects of public health and sustainable development that permeate the implementation of public policies appropriate to the promotion of health and quality of life in the scenaries of contemporary communities. The relevance of this study contributes to the reflection,
\end{abstract}

\footnotetext{
Artigo Original: Recebido em 30/09/2020 - Aprovado em 23/10/2020.

${ }^{1}$ Mestre em Desenvolvimento Territorial Sustentável pela Universidade Federal do Paraná (UFPR), Setor Litoral. Graduada em Fisioterapia. Filiada ao grupo Laboratório de Análise de Redes/UFPR. E-mail: dani.cardeal@hotmail.com (autor correspondente)

${ }^{2}$ Doutor em Ciência Política pela Universidade Federal do Rio Grande do Sul (UFRGS). Professor do Curso de Administração Pública na Universidade Federal do Paraná (UFPR), Setor Litoral. Filiado ao grupo de pesquisa Laboratório de Análise de Redes (UFPR).E-mail: augustoclemente@ufpr.br

${ }^{3}$ Mestranda em Políticas Públicas pela Universidade Federal do Pampa (UNIPAMPA), campus São Borja. Graduada em Administração (Universidade Regional Integrada do Alto Uruguai e das Missões - URI). Filiada ao grupo de pesquisa Laboratório de Análise de Redes (UFPR).E-mail: marcia.r.c.perini@gmail.com

${ }^{4}$ Doutoranda em Ciência Política pela Universidade Federal do Rio Grande do Sul (UFRGS). Mestre em Políticas Públicas pela Universidade Federal do Pampa (UNIPAMPA), campus São Borja. Graduada em Ciência Política (UNIPAMPA). Filiada ao grupo de pesquisa Laboratório de Análise de Redes (UFPR).E-mail: irisnabolotnyj@gmail.com

${ }_{5}^{5}$ Mestranda em Políticas Públicas pela Universidade Federal do Pampa (UNIPAMPA), campus São Borja. Graduada em Serviço Social (UNIPAMPA). Filiada ao grupo de pesquisa Laboratório de Análise de Redes (UFPR). E-mail: caroline.p.x@hotmail.com
} 
motivation and mobilization of practical and theoretical subsidies about cities, motivating the appropriate use of economic resources combining well-being of the inhabitants' lives and less pollutant emissions, which corresponds to the implementation of public policies aimed at the needs of improving the population's life.

Keywords: Information and Communication Technologies (ICTs); Sustainable development; Public Health Promotion.

\section{Introdução}

A partir do século XX, com a ascensão das novas tecnologias, as cidades experimentaram mudanças significativas. A interação cada vez mais rápida das informações obtidas modificou as redes locais, assim como também as relações entre os atores da esfera pública com os setores da sociedade civil. Nesse sentido, as Tecnologias da Informação e da Comunicação (TICs) criaram indicadores de impacto sobre as cidades motivando condições urbanas mais adequadas, humanizadas, saudáveis e sustentáveis que se caracterizam pela terminologia "cidades inteligentes" (smart cities) (ANDERLE, 2017).

As condições socioambientais, socioculturais e sociopolíticas se tornaram pilares fundamentais que exprimem um novo modelo de desenvolvimento nas cidades, incorporando o adjetivo "sustentável" às pautas de discussões em prol de cidades mais conectadas com a fluidez da vida coletiva e com a função de cada serviço público sobre a vida da população, dando especial atenção aos fatores que propulsionam a saúde pública, o bem-estar e a qualidade de vida das pessoas. No entanto, estas reflexões parecem ainda pouco exploradas e incipientes no cenário prático das políticas públicas locais.

Dessa maneira, este ensaio tem como objetivo explanar sobre os elos entre as cidades inteligentes, o desenvolvimento sustentável e a saúde, articulando o papel do Estado por meio das políticas públicas. Segundo Costa e Hardagh (2018), a efetivação das políticas públicas passa pelo fortalecimento dos interesses entre as entidades públicas e privadas que visam uma maior coesão social para sua implementação através da tecnologia. A contribuição da tecnologia nas cidades, desenvolve a cidadania e incentiva um cenário disruptivo, que permite compreender se a cidade está sendo construída para controlar, ou para promover a convivência coletiva de maneira dinâmica.

Sendo assim, este artigo está organizado da seguinte forma, além desta introdução. Na seção 2, discutiremos o contexto teórico no qual está situado o conceito de cidades inteligentes. Em seguida, na seção 3, a tríade conceitual de cidades inteligentes, sustentabilidade e saúde serão aprofundados, observando e apontando esta conjunção como promissora. Na seção 4, explicaremos como as políticas públicas voltadas à sustentabilidade e à saúde pública nas cidades são fatores importantes em prol do desenvolvimento local. Por último, algumas considerações finais e referências serão registradas, respectivamente.

\section{Cidades Inteligentes}

Historicamente, a sociedade vivencia mudanças, especialmente nesse milênio marcado pelo crescimento acelerado da alta tecnologia, a qual possibilita o gerenciamento da capacidade de distribuir informações. Tal capacidade desencadeou uma verdadeira revolução de informações e aprendizagens, influenciando de forma contundente a maneira como as cidades e as sociedades se organizam.

Pensar nessa organização urbana pode se tornar um desafio quando se considera as necessidades de bem-estar da população. A ascensão da sociedade civil organizada, propriamente dita, se dá a partir do século XVIII, com o êxodo rural e o desenvolvimento urbano movimentando as populações do campo para a centros das cidades. No século XIX, porém, essas mudanças atingem o seu ápice, perdurando mais um século de aperfeiçoamento sobre as condições de vida menos 
insalubres e minimamente harmônicas da população em expansão (SANTOS, 1993).

Neste sentido, a Revolução Industrial demarca o período de importantes transformações. Chiavenato (2014) relata que "ao mesmo tempo em que a intensa migração de mão de obra se deslocava dos campos agrícolas para os centros industriais, surgia um surto acelerado e desorganizado de urbanização" (p. 41) por meio do inchaço das cidades, que trouxe a eminente necessidade de administração pública.

Tendo em vista todas as mudanças acarretadas pela urbanização, Weiss, Bernardes e Consoni (2015), indicam algumas perdas de funcionalidades fundamentais que influenciaram fortemente a qualidade de vida das pessoas. Para esses autores, as principais deficiências estão "na gestão de resíduos; escassez, desperdícios e má gestão dos recursos naturais; restrições nos sistemas de saúde, educação e segurança pública; limitações nos sistemas de mobilidade urbana e de transportes; obsolescência e encurtamento do ciclo de vida das infraestruturas públicas" (p. 3).

As transformações acarretadas desde a Revolução Industrial são inúmeras, em diferentes campos, seja na nova concepção de trabalho, que reflete na estrutura social e comercial desde à época até a atualidade, seja nas mudanças de ordem econômica, política e social, de forma muito mais rápida do que o milênio anterior. Em meio a essas alterações, as políticas públicas também foram afetadas por abarcarem as tomadas de decisões resolutivas sobre os problemas públicos, em diversos setores, tais como saúde, assistência, habitação, educação, emprego, renda fiscal e monetária, ciência, tecnologia, entre outros (CHIAVENATO, 2014).

Em vista disso, na busca por formular políticas públicas capazes de atender aos diversos problemas públicos são evidentes as contribuições da tecnologia, especialmente sobre as cidades. Weiss, Bernardes e Consoni (2015), consideram que a inclusão de tecnologias deve promover:

maior eficiência energética e otimização na produção de bens e serviços; sistemas inteligentes para $\mathrm{o}$ monitoramento $\mathrm{e}$ gerenciamento das infraestruturas urbanas e antecipação a acidentes naturais; soluções de colaboração e redes sociais; sistemas integrados para a gestão de ativos; sistemas especializados de atenção à saúde e educação que permitem a interação com os atores por intermédio da internet; sistemas, métodos e práticas para o gerenciamento integrado de serviços de qualquer natureza; sistemas para $\mathrm{o}$ tratamento de grandes volumes de dados estruturados e não estruturados; sistemas de georreferenciamento; aplicações inteligentes embarcadas em toda sorte de bens; tecnologias de identificação por radiofrequência e etiquetas digitais colocadas em produtos e cargas, otimizando os processos logísticos e as transações comerciais; sensores e sistemas de inteligência artificial que percebem e respondem rapidamente a eventos ocorridos no mundo físico, desencadeando processos digitais que passam a ter consequências cada vez mais imediatas e significativas no mundo, conectando pessoas, empresas e poder público a qualquer tempo e em qualquer lugar (WEISS; BERNARDES; CONSONI, 2015, p. 3).

Desse modo, o termo "cidades inteligentes" deriva do inglês "smart cities" e significa o nível mais elevado entre a convergência tecnológica, a gestão de cidades, a qualidade de vida e a competitividade econômica (STRAPAZZON, 2009). O surgimento da terminologia aparece, de acordo com Strapazzon (2009), em 1999, com a premiação da cidade de Singapura como cidade inteligente, sendo, por isso, na sequência analisada com maior profundidade e inspirando alternativas também para cidades de médio e pequeno porte.

Gomes e Paliologo (2017), mostram que o termo teve a sua primeira atribuição a partir da terminologia de "crescimento inteligente" (smart growth), caracterizado como um movimento que propunha o uso das TICs incorporado ao planejamento urbano. Os autores expõem ainda que esse sistema passou a ser testado por grandes organizações do ramo da tecnologia, a partir dos anos 2000. Contemporaneamente, as organizações de tecnologia encontraram, na administração pública, clientes potenciais, iniciando aplicações nas cidades já existentes (GOMES; PALIOLOGO, 2017).

Além disso, outra definição, mais abrangente, a respeito das cidades inteligentes é ditada por Cury e Marques (2017), que a qualificam como "áreas com alta capacidade de aprendizagem e inovação construídas com base na criatividade de sua comunidade, de suas instituições e de sua 
infraestrutura digital para a comunicação e gestão do conhecimento" (CURY; MARQUES, 2017, p. 106).

Sob outra perspectiva, Paulo Freire (2001) define que a cidade também educa. E:

\begin{abstract}
enquanto educadora, a Cidade é também educanda. Muito de sua tarefa educativa implica a nossa posição política e, obviamente, a maneira como exerçamos o poder na Cidade e o sonho ou a utopia de que embebamos a política, a serviço de que e de quem a fazemos. A política dos gastos públicos, a política cultural e educacional, a política de saúde, a dos transportes, a do lazer (FREIRE, 2001, p. 13).
\end{abstract}

Desse modo, há uma intencionalidade eminente em observar como as políticas públicas para as cidades inteligentes podem apresentar contribuições efetivas às problemáticas atuais, tais como: a geração de conhecimentos que eduquem com foco no desenvolvimento, além da saúde, da segurança pública, dos resíduos urbanos, da mobilidade, entre tantos outros (FREIRE, 2001).

Caragliu, Del Bo, Nijkamp (2011), diferentemente das definições dos anos 90, que tiveram foco na infraestrutura de informação e comunicação, argumentam que atualmente aquelas acepções não são suficientes.

Acreditamos que uma cidade é inteligente quando investimentos em capital humano e social, (transporte), moderna infraestrutura de comunicação (TIC), alimentam o crescimento econômico sustentável e alta qualidade de vida, com uma gestão inteligente dos recursos naturais, através de governança participatica . (CARAGLIU; DEL BO; NIJKAMP, 2011, p. 6, tradução nossa).

Tal reflexão torna-se relevante, pois a disponibilidade da tecnologia não deve ser o único fator para se considerar a inteligência de um espaço urbano, pelo contrário, a importância da qualidade dessa disponibilidade, o papel do capital humano e a educação (com a formação de habilidades para as vivências nesses espaços), assim como também os aspectos ambientais como impulsionadores de um crescimento urbano saudável e sustentável são princípios norteadores ainda mais importantes para a arena política. A seguir, aprofundaremos a temática trazendo pontos mais específicos sobre a sustentabilidade e a saúde pública.
3 Sustentabilidade e Saúde Pública: uma relação promissora em prol das cidades inteligentes

Pensar sobre uma perspectiva econômica é certamente uma abordagem interessante para se construir argumentos sobre as cidades inteligentes. Entretanto, esta, deve estar atrelada a perspectiva de desenvolvimento pleno, integrando os aspectos que tangenciam a vida da população. Este foco distinto de progresso, abarca muitas vezes um olhar sobre outras especificidades, não somente propostas por meio do lucro financeiro, dos valores monetários e das aquisições materiais. $O$ conceito de "desenvolvimento sustentável", segundo Sachs (1986), embora muitas vezes tenha soado polissêmico, nasce a partir de um foco mais amplo, que compreende uma análise sobre os resultados sustentáveis das cidades (SACHS, 1986; SEN, 2000; VEIGA, 2014). Em 1960, surge um novo olhar sobre as agendas de discussões, com a tríade ambienteeconomia-humanidade, que tinham como objetivo vencer os agravos impactos ao meio ambiente pelo mundo (CARSON, 1962).

As agendas ambientais discutidas pela Organização das Nações Unidas (ONU), desde o marco histórico da Agenda 21, na Rio+20/2012, anexou a saúde aos condicionantes socioambientais, até a recente Agenda 2030/2015, onde foram estabelecidos os 17 Objetivos do Desenvolvimento Sustentável (ODS) e 169 metas globais reafirmavam o compromisso de escala e ambição universal. Esta última agenda ambiental se constrói sobre o legado dos 8 Objetivos do Desenvolvimento do Milênio/2000 (ODM) com o esforço na execução dos alvos e metas que não puderam ser alcançadas (UNITED NATIONS, 2020). Muitos destes objetivos conectam um ambiente ecologicamente equilibrado, com o foco na promoção da saúde e do bem-estar dos indivíduos, promovendo o cuidado, fatores sociais cruciais para o dinamismo das cidades.

Alguns desses avanços tecnológicos possibilitaram um novo formato social às cidades, facilitando o cotidiano e o conforto das pessoas. Em 2007, se tinha mais da metade da população mundial vivendo em cidades e se espera uma projeção de mais $60 \%$ em 2030. Neste sentido, as cidades apresentam em média $70 \%$ das emissões de gás carbono no mundo e mais de $60 \%$ dos usos de recursos disponíveis. Entretanto, com foco nos 
grandes espaços urbanos, nos impactos dessa transformação e no meio ambiente, a ONU propôs em seu ODS 11 - Cidades e Comunidades Sustentáveis - tornar as cidades e os espaços urbanos inclusivos, seguros, resilientes e sustentáveis. Vários pontos foram incluídos nessa meta: aumentar a urbanização inclusiva e sustentável; urbanizar favelas e garantir saneamento básico para a toda sociedade; acesso universal a espaços públicos sustentáveis, saudáveis, verdes, etc.; dentre outros. Tudo isso aliado ao conceito das cidades inteligentes (smart cities) (NACIONES UNIDAS, 2020; REDE BRASILEIRA DE CIDADES INTELIGENTES E HUMANAS, 2020).

Atualmente, o adjetivo "sustentável” já se faz disseminado em um cenário que requer mudanças substanciais sobre as dimensões ecológica, econômica e social. Baseado no entendimento clássico de que se deve satisfazer "as necessidades do presente sem comprometer a capacidade das gerações futuras em satisfazer suas próprias necessidades" (BRUNDTLAND, 1987, p. 46), as cidades precisam adequarem-se à vida moderna, sendo incentivadas a melhores ações sobre os serviços de saúde que resultem no bem-estar da população. Souza, Freitas e Gonçalves (2018), defendem que, com um modo de vida sustentável em seus meios de produção e consumo, será possível garantir que outras gerações possam desfrutar dos recursos que se tem disponível hoje.

Essa concepção ampliada sobre a saúde, muito mais do que uma proposta bem-estar global anunciada pela Organização Mundial de Saúde (OMS), propõe "um completo estado de bem-estar físico, mental e social, e não apenas a ausência de doença ou enfermidade" (WHO, 1946, p. 1), e promove a garantia de provisão de um conjunto de benefícios e bens que abrangem a esfera social. É estratégico pensar nesta abordagem frente as políticas nacionais, pois é no espaço territorial das cidades que as políticas públicas realmente se efetivam (GADELHA et al., 2011). Poletto, Klenubing e Bossi (2019) corroboram essa posição, defendendo que a inter-relação do ser humano necessita estar situada no centro desta análise: devese ter o cuidado com a promoção da qualidade de vida e bem-estar da população urbana.

Com esses conceitos bem estabelecidos e explorados no panorama mundial das discussões, nos chama atenção a necessidade de um modelo de desenvolvimento mais adequado ao cenário atual (MALDANER et al, 2017). Neste sentido, um formato mais saudável e sustentável de cidades pode contribuir para melhoria de vida dos habitantes. $\mathrm{Na}$ próxima seção, apontaremos a relação desses conceitos, dessa vez, com as políticas públicas para as cidades.

\section{Políticas Públicas, Saúde Pública e Sustentabilidade nas Cidades Inteligentes}

A maior consequência da Revolução Industrial foi a urbanização. Porém, foram inúmeros os desafios para a administração pública intencionar políticas com o foco nas cidades inteligentes. As diferentes formas de desenvolver as cidades, gerar energia limpa, conscientizar o cuidado no uso dos recursos naturais, promover a mobilidade urbana, a educação e a saúde, mostram como estes temas são emergenciais, de extrema prioridade, para os promotores das políticas públicas, envolvendo não apenas os governos, mas principalmente as necessidades de cada cidade e população peculiar.

As mudanças na conjuntura das últimas décadas apontam para a valorização e a abrangência das políticas públicas pelos estudos acadêmicos. Muitos motivos possibilitaram essa ascensão, um deles é a adoção, por parte dos governos, de políticas restritivas de gastos, economia de recursos, e promoção de políticas sociais com ênfase na saúde (SOUZA, 2007).

Neste sentido, Secchi (2016) conceitua as políticas públicas, relembrando que para o bom entendimento deste campo de estudo emerge a clareza sobre dois conceitos fundamentais: o problema público e a política pública. O problema, é o ponto de partida da análise e pode ser entendido como a distância entre o status quo e a situação desejada para determinada realidade pública. Já a política pública, é uma diretriz que foi elaborada para o enfrentamento do problema público.

Assim, a promulgação da Constituição Federal Brasileira, de 1988, popularmente conhecida como Carta Cidadã, trouxe a regulamentação de dois artigos, 182 e 183 pela Lei 10.257/2001, que institui o Estatuto das Cidades, estabelecendo diretrizes 
acerca da política urbana brasileira que representam dois marcos na promoção do desenvolvimento urbano do país (BRASIL, 2001). A partir desses importantes avanços, como possível resposta aos problemas públicos urbanos, inicia-se a promoção de estudos sobre o papel das cidades inteligentes e sustentáveis em prol do planejamento urbano.

Embora as gestões municipais estejam fortemente vinculadas ao interesse e a adesão das iniciativas e dos projetos no âmbito, torna-se relevante uma visão nacional sobre o tema. Dessa maneira, algumas diretrizes e linhas de financiamento para a implementação de cidades inteligentes pelas administrações públicas municipais estão em construção no Brasil. A atuação do Ministério da Ciência, Tecnologia e Inovação (MCTI), Ministério das Comunicações (MCOM) e do Ministério do Desenvolvimento Regional (MDR), têm apontado alguns caminhos para essa estruturação. Aqui, podemos traçar referências salutares à formulação e a implementação de políticas públicas direcionadas às cidades, são elas:

- Portaria $n^{\circ} 376$, de 19 de agosto de 2011, alterada pela Portaria $n^{\circ} 186$, de 29 de março de 2012 estabelece o Programa Cidades Digitais (BRASIL, 2011).

- Decreto 9.612/2018 - dispõe sobre políticas públicas de telecomunicações, especificando a substituição do Programa Cidades Digitais, pelo Programa de Cidades Inteligentes (BRASIL, 2018).

- Decreto 9.854/2019 - institui o Plano Nacional de Internet das Coisas e está sendo desenvolvido em consonância com o Programa de Cidades Inteligentes (BRASIL, 2019).

Sendo um tema de grande complexidade, atualmente os ministérios MCTI e MDR, por meio da elaboração da Carta Brasileira para Cidades Inteligentes, traçam, em cooperação uma estratégia nacional, a estruturação do desenvolvimento de cidades inteligentes e sustentáveis a partir de diversas ações que abrangem quatro câmaras interministeriais: a) Cidades 4.0; b) Saúde 4.0; c) Rural 4.0 e d) Indústrias 4.0 (BRASIL, 2020).

Dentre as pautas focalizadas estão os critérios de seleção de indicadores para o Brasil que promovem o modelo de maturidade para as cidades inteligentes, considerando: i) adequação à realidade dos municípios brasileiros; ii) atendimento à especificação conceitual do modelo de maturidade da UIT (União Internacional de Telecomunicações); iii) aderência à agenda 2030 (ODS); iv) disponibilidade preferencial em fontes de dados secundários; v) efetiva contribuição para o planejamento de ações e estratégias na construção de políticas públicas para cidade inteligente; vi) serviços que podem ser ampliados pelo uso de TIC; vii) aderência à Carta Brasileira de Cidades Inteligentes (ROMANO, 2020).

Segundo Romano (2020), a elaboração dos indicadores do modelo de maturidade brasileiro aponta indicadores já dispostos pela União Internacional de Telecomunicações; pelo Cities in Motion Index; pelo Programa para Cidades Inteligentes e Sustentáveis; pelos Objetivos de Desenvolvimento Sustentável (ODS); pela Agência Brasileira de Desenvolvimento Industrial; e também as ISO: 37120 - Sustainable Cities and Communities - Indicators for Urban Services and Quality of Life; 37122 - Sustainable Cities and Communities Indicators for Smart Cities, e 37123 - Sustainable Cities and Communities -Indicators for Resilient Cities; além de 16 estudos acadêmicos nacionais e internacionais.

Diante do que pode ser observado, a caminhada brasileira em busca pela implementação das cidades inteligentes e sustentáveis ainda é incipiente e desafiadora, especialmente pelas desigualdades e problemas públicos que marcam o panorama das cidades.

Compreendendo estas restrições, é relevante refletir as suas contribuições sobre a sustentabilidade. Segundo Strapazzon (2009), tendo como referência o documento elaborado pelo projeto europeu de cidades inteligentes (smart cities), para ser considerada inteligente a cidade necessita atender com um desempenho adequado de seis aspectos. As expressões definem as cidades como espaços vitais adequados, bons lugares para o desenvolvimento econômico, ou seja, são imprescindíveis no momento da elaboração de políticas públicas, conforme demonstra o quadro 1 . 
Quadro 1 - Quesitos de desempenho para espaços vitais e bons espaços de desenvolvimento econômico

\begin{tabular}{|c|c|}
\hline Quesito & Descrição \\
\hline a) Economia & $\begin{array}{l}\text { inteligência econômica: gerar capacidade de inovação, competitividade, } \\
\text { empreendedorismo, flexibilidade nas relações de trabalho, influenciar } \\
\text { positivamente nas taxas de desemprego e aprimorar o sistema de transporte } \\
\text { público; }\end{array}$ \\
\hline b) Sociedade & $\begin{array}{l}\text { habitantes inteligentes: população local com cultura cosmopolita, o que, entre } \\
\text { outros, exige domínio de língua estrangeira, participação dos assuntos públicos, } \\
\text { educação formal adequada, afinidade com a educação continuada, boa cultura } \\
\text { geral, bom índice de livros lidos por habitante, tolerância étnica, atuação em } \\
\text { atividades voluntárias, participação nas eleições; }\end{array}$ \\
\hline c) Governo & $\begin{array}{l}\text { governo inteligente: um sistema de gestão pública participativo, gerador de } \\
\text { serviços públicos e sociais, transparente e dotado de perspectivas estratégicas; }\end{array}$ \\
\hline d) Mobilidade & $\begin{array}{l}\text { inteligente sistema logístico e de transporte de pessoas: meios eficientes de } \\
\text { acessibilidade local e internacional, um sistema de transporte sustentável - não } \\
\text { agressivo ao meio ambiente - e ter amplo acesso à internet; }\end{array}$ \\
\hline e) Meio Ambiente & $\begin{array}{l}\text { gestão inteligente dos recursos naturais: a cidade deve ter boa gestão dos espaços } \\
\text { verdes, ter programas de reciclagem e proteção ambiental, ter programa } \\
\text { sustentável de gestão da água, da energia, do lixo e da poluição, e fazer bom uso } \\
\text { de seus espaços naturais, de modo a torná-los atrativos; }\end{array}$ \\
\hline f) Qualidade de Vida & $\begin{array}{l}\text { facilidades culturais: boa educação formal, bom sistema de saúde e segurança } \\
\text { individual, as características das moradias devem ser sustentáveis e agradáveis, } \\
\text { deve ter opções para atrações turísticas, e ter um bom nível de coesão social, } \\
\text { isto é, com programa de percepção do risco social, decorrentes dos altos níveis } \\
\text { de pobreza. }\end{array}$ \\
\hline
\end{tabular}

FONTE: Adaptado de STRAPAZZON (2009, p. 95).

Para Mahler (2016), existem sete pontos chaves indicados pelo Green City Index para uma cidade se tornar sustentável: i) bom governo, ii) soluções integradas, iii) valorização da saúde da população, iv) incentivo à participação do cidadão, v) bom uso da tecnologia, vi) equilíbrio entre crescimento econômico e preservação do meio ambiente, e vii) iniciativas de entidades não governamentais.

Neste cenário, as TICs surgem como instrumento principal na criação de inovações que acarretam melhorias nos contextos citados assumindo o papel de ferramentas de gestão das infraestruturas, bem como auxiliando no atendimento das necessidades da população. Quando direcionamos o olhar para o atual momento brasileiro, os desafios para tais implementações são ainda maiores, dadas as condições econômicas, sociais e políticas evidenciadas nas cidades.

Considerando essas inquietações no âmbito das políticas públicas, torna-se essencial equilibrar as alternativas disponíveis ao se implementar, por exemplo, políticas de incentivo a eficiência energética e a sua geração descentralizada, com a inserção do excedente na rede das concessionárias (FERREIRA et al., 2015).

Dessa maneira, compreende-se que ao pensar políticas públicas para cidades inteligentes estejam relacionadas a geração de ambientes saudáveis e sustentáveis. A relação entre os ecossistemas locais, pois a busca pela qualidade de vida da sociedade é um dos maiores desafios da atualidade. Ao estruturar ações para sanar esses desafios, é imprescindível entender sobre os campos social, econômico e ambiental que estão diretamente ligados ao cenário das cidades inteligentes (CURY; MARQUES, 2017).

Tendo em vista o tripé da sustentabilidade (ambiental, econômico e social), pode-se pautar que a inteligência da cidade abrange as habilidades e as capacidades produtivas locais, considerando igualmente a cultura e as atividades econômicas, de forma a melhor valer-se de suas potencialidades. Perpassa, além disso, a descoberta de novas habilidades, que de fato auxiliarão na geração de empregos e de investimentos, tanto públicos como privados, com foco no aprimoramento da qualidade de 
vida e no alcance do desenvolvimento para o maior número de pessoas possível (CAMARGO, 2015).

A consolidação das cidades inteligentes deve ser elaborada e formulada por meio de políticas públicas que norteiem a sua efetivação de modo a fortalecêla, promovendo o interesse da sociedade articulada com entes públicos e organizações privadas, trazendo, assim, uma maior coesão social na sua implementação.

Contudo, é necessário analisar se as práticas e apelos tecnológicos das cidades inteligentes contribuem para a realidade de uma cidade saudável, conjecturando os caminhos alternativos na educação do cidadão a partir de uma visão mais clara das contribuições efetivas no cenário das cidades inteligentes, assim como também aperfeiçoando o seu aparato tecnológico, denominado pelos autores como apelo "tech". Dessa forma, é imprescindível observar aspectos como a obsolescência produtiva, o hibridismo humano-tecnológico e a conexão digital que, por um lado, promove o desenvolvimento, mas, por outro, excluem pessoas iletradas tecnologicamente. A contribuição da tecnologia na cidade e na cidadania em um cenário disruptivo, permite questionar se a cidade está sendo construída para controlar, ou para promover, a convivência de maneira saudável e inclusiva (COSTA; HARDAGH, 2018).

\section{Considerações Finais}

Diante do cenário atual, as discussões sobre a efetivação das políticas públicas são anseios em prol de ambientes mais saudáveis e sustentáveis. Idealizar novos paradigmas sobre essa construção, certamente pauta as agendas ambientais difundidas para a elaboração de cidades inteligentes. Desse modo, incentivar estudos que sintetizem a relação entre os conceitos de sustentabilidade e de saúde focados nos resultados das ações do Estado na vida das pessoas é bastante promissor.

Motivar os ainda incipientes estudos sobre o uso das TICs na geração de cidades onde as redes e os serviços tradicionais se tornam mais eficientes para o benefício de seus habitantes. Uma cidade inteligente supera o melhor uso de recursos e menos emissões de poluentes para um cenário mais abrangente pautado em parâmetros sustentáveis que, certamente, se articulam com a efetivação de políticas públicas adequadas às necessidades das populações em atender suas demandas de saúde, sociais, econômicas.

Dessa maneira, este trabalho evidenciou o pensamento analítico comum de vários autores, citados no sub título anterior, que sintetizam um projeto para uma cidade inteligente, ultrapassando um pensar sobre as práticas das TICs e integrando as redes efetivas de transporte urbano, melhor abastecimento de água e instalações de eliminação de resíduos e promovendo efeitos sustentáveis de geração de energia. Portanto, não se trata de fatores isolados, mas integrados e dinâmicos. Tudo isso permeia uma administração pública mais interativa e ágil, em que a segurança e o desenvolvimento local promovam uma melhor qualidade de vida por meio das políticas públicas.

O desafio já foi lançado. O envolvimento do público, da indústria e de outros grupos interessados para desenvolver soluções inovadoras de governança nas cidades brasileiras pode ser alcançado através de mais estudos como este, que evidenciem abordagens viáveis para a implementação das cidades inteligentes. Esse trabalho sintetizou, finalmente, que, assim como os resultados demonstrados, o compartilhamento de conhecimento e de dados exprimem um caminho factível nessa jornada. Muito mais precisa ser feito e mais estudos desenvolvidos alicerçando desenvolver e implementar soluções integradas em prol das cidades inteligentes saudáveis e sustentáveis devem ser propostos.

\section{Referência}

ANDERLE, D. F. Modelo de conhecimento para representação semântica de smart cities com foco nas pessoas. 2017. 240 f. Tese (Doutorado em Engenharia e Gestão do Conhecimento) - Centro Tecnológico, Universidade Federal de Santa Catarina, Florianópolis, 2017.

BRASIL. Ministério do Desenvolvimento Regional. Carta Brasileira para Cidades Inteligentes. Brasília, 2020. Disponível em: $<$ https://www.gov.br/mdr/pt-

br/assuntos/desenvolvimento-regional/projetoandus/carta-brasileira-para-cidades-inteligentes> 
BRASIL. Lei $\mathrm{n}^{\circ}$ 10.257, de 10 de julho de 2001. Regulamenta os arts. 182 e 183 da Constituição Federal, estabelece diretrizes gerais da política urbana e dá outras providências. Portal da Legislação. Brasília, 2001. Disponível em: $<$ http://planalto.gov.br/ccivil_03/leis/leis_2001/1 10257.htm>

BRASIL. Portaria $\mathrm{n}^{\circ}$ 376, de 19 de agosto de 2011, alterada pela Portaria $\mathrm{n}^{\circ} 186$, de 29 de março de 2012 - estabelece o Programa Cidades Digitais. Diário oficial da União. Brasília, DF, 29 mar. 2012. Disponível em: $<$ https://pesquisa.in.gov.br/imprensa/servlet/INPDF Viewer?jornal $=1$ \&pagina $=117 \&$ data $=29 / 03 / 2012 \&$ captchafield $=$ firstAccess $>$

BRASIL. Decreto $\mathrm{n}^{\circ}$ 9.612, de 17 de dezembro de 2018. Dispõe sobre políticas públicas de telecomunicações. Portal da Legislação. Brasília, $2018 . \quad$ Disponível em: $<\mathrm{http}$ //www.planalto.gov.br/ccivil_03/_Ato20152018/2018/Decreto/D9612.htm>

BRASIL. Decreto no 9.854, de 25 de junho de 2019. Institui o Plano Nacional de Internet das Coisas e dispõe sobre a Câmara de Gestão e Acompanhamento do Desenvolvimento de Sistemas de Comunicação Máquina a Máquina e Internet das Coisas. Portal da Legislação. Brasília, 2019. Disponível em:

$<$ http://www.planalto.gov.br/ccivil_03/_ato20192022/2019/decreto/D9854.htm>

BRUNDTLAND, G. H. Nosso futuro comum. Rio de Janeiro: FGV, 1987.

CAMARGO, A. Depoimento testimonial. Cidades Inteligentes e mobilidade urbana. Cadernos FGV Projetos, ano 10, n. 24, p. 14-19, out. 2015.

CARAGLIU, A.; DEL BO, C.; NIJKAMP, P. Smart Cities in Europe. Journal of Urban Technology, v. 18 , n. 2, p. 65-82, 2011.

CARSON, R. Primavera Silenciosa. 2. ed. São Paulo: Portico, 1962.

CHIAVENATO, I. Introdução à teoria geral da administração. $9^{a}$ ed. São Paulo: Manole, 2014.

COSTA M. M. A. F. F. da; HARDAGH, C. C. Cidades inteligentes são cidades saudáveis? Labor E Engenho, v. 12, n. 4, p. 525-532, 2018.

CURY, M. J. F.; MARQUES, J. A. L. F. A cidade inteligente: uma reterritorialização. Redes, v. 22, n. 1, p. 102-117, 2017.

FERREIRA, M. L.; AGUIAR, A. O.; CORTESE, T. T. P.; KNIESS, C. T.; QUARESMA, C. C.; PASCHOALIN FILHO, J. A. Cidades inteligentes e sustentáveis: problemas e desafios. 2015.
Disponível em: $<$ https://www.researchgate.net/publication/2832978 22_Cidades_inteligentes_e_sustentaveis_problemas _e_desafios $>$. Acesso em: $17 \mathrm{de}$ fev. de 2020.

FREIRE, P. Política e educação: ensaios. 5. ed. São Paulo: Cortez, 2001.

GADELHA, Carlos Augusto Grabois; MACHADO, Cristiani Vieira; LIMA, Luciana Dias de; BAPTISTA, Tatiana Wargas de Faria. Saúde e territorialização na perspectiva do desenvolvimento. Ciência e Saúde Coletiva, v. 6, n. 6, p. 3003-3016, 2011.

GOMES, D. M.; PALIOLOGO, N. A. Direito à cidade e políticas públicas para a smart city. Revista de Direito Urbanístico, Cidade e Alteridade, v. 3, n. 1, p. 19-35, jan./jun. 2017.

MAHLER, E. M. M. Cidades sustentáveis no contexto brasileiro. Caderno de Gestão Pública. v. 8, n. 5, 2016.

MALDANER, Tainara Piontkoski; SANTOS, Bruna Letícia; WEINERT, Luciana Vieira Castilho; WEINERT, Wagner Rodrigo. Saúde, Sustentabilidade e Infância: reflexões sobre uma perspectiva ambiental. Divers@, Matinhos, v. 10, n. 2, p. 60-68, jul./dez. 2017.

NACIONES UNIDAS. Objetivos de Desarrollo Sostenible: Objetivo 11 - Lograr que las ciudades sean más inclusivas, seguras, resilientes y sostenibles. 2020. Disponível em: https://www.un.org/sustainabledevelopment/es/cities/

POLETTO, E. C.; KLENUBING, N. V.; NELI, S. B. Ferramentas pedagógicas para desenvolvimento da educação não formal por profissionais da vigilância em saúde de Matinhos - Pr. Divers@, Matinhos, v. 12, n. 1, p. 11-20, jan./jun. 2019.

REDE BRASILEIRA DE CIDADES INTELIGENTES E HUMANAS. ODS 11: cidades sustentáveis e sua relação com Smart Cities. 2020. Disponível em:

https://redebrasileira.org/materias/3221/ods-11cidades-sustentaveis-e-sua-relacao-com-smartcities>

ROMANO, R. R. Connected Smart Cities e Mobility Digital Xperience 2020. 2020. (24m34s). Disponível em: $<$ https://www.youtube.com/watch?v=MkBc9s4TyB E>. Acesso em: 10 set. 2020.

SACHS, I. Ecodesenvolvimento crescer sem destruir. Terra dos Homens. São Paulo: Editora Vértice, 1986.

SANTOS, M. A urbanização brasileira. São Paulo: HUCITEC, 1993. 
SECCHI, Leonardo. Análise de políticas públicas: diagnóstico de problemas, recomendação de soluções. São Paulo, Cengage Learning, 2016.

SEN, A. Desenvolvimento como Liberdade. São Paulo: Companhia das Letras, 2000.

SOUZA, C. Estado da arte da Pesquisa em Políticas Públicas. In: HOCHMAN, G.; ARRETCHE, M.; MARQUES, E. Políticas Públicas no Brasil. Rio de Janeiro: Editora Fiocruz, 2007. p. 65-86.

SOUZA, M. L. DE; FREITAS, D. A. F. DE; GONÇALVES, C. S. Biodegradabilidade para a produção de energia renovável. Divers@, Matinhos, v. 11, n. 1, p. 26-38, jan./jun. 2018.

STRAPAZZON, C. L. Convergência tecnológica nas políticas urbanas: pequenas e médias “cidades inteligentes". 2009. Disponível em: $<$ https://edisciplinas.usp.br/pluginfile.php/4315777/ mod_resource/content/1/Convergencia $\% 20$ Tecnolo gia $\% 20-\% 20$ Smart $\% 20$ Cities.pdf>
UNITED NATIONS. Make the SDGS a Reality.

$2020 . \quad$ Disponível em:

$<$ https://sustainabledevelopment.un.org/>

VEIGA, J. E. O âmago da sustentabilidade. Estudos Avançados, v. 82, p. 7-23, 2014.

WEISS, A. C.; BERNARDES, R. C.; CONSONI, F. L. Cidades inteligentes como nova prática para o gerenciamento dos serviços e infraestruturas urbanos: a experiência da cidade de Porto Alegre. urbe, Revista Brasileira de Gestão Urbana, Curitiba, v. 7, n. 3, p. 310-324, dez. 2015.

WORLD HEALTH ORGANIZATION - WHO. Constitution of the World Health Organization. Basic Documents. Geneva: World Health Organization, 1946. 